\title{
Reducing oxidative stress may be important for treating pirarubicin-induced cardiotoxicity with schisandrin B
}

\author{
HENG TANG $^{1 *}$, JUNHAO ZHAO $^{2 *}$, RUI FENG $^{1}$, PENG PU $^{1}$ and LI WEN ${ }^{1}$ \\ ${ }^{1}$ Department of Cardiology, The First Affiliated Hospital of Chongqing Medical University, Chongqing 400042; \\ ${ }^{2}$ The First Clinical College, Jinyun Mountain Campus of Chongqing Medical University, Chongqing 401331, P.R. China
}

Received August 19, 2021; Accepted November 8, 2021

DOI: $10.3892 /$ etm.2021.10991

\begin{abstract}
The cardiotoxicity of pirarubicin (THP) seriously affects its clinical application, which cannot be ignored. The antioxidant effect of schisandrin $\mathrm{B}(\mathrm{SchB})$ has been extensively reported in the context of dietotherapy. However, whether this antioxidant effect can protect the heart from THP damage remains unknown. The aim of the present study was to investigate whether the antioxidant effect of SchB can antagonize the cardiotoxicity of THP. Changes in electrocardiogram (ECG), echocardiography and serum lactate dehydrogenase, brain natriuretic peptide, creatine kinase $\mathrm{MB}$ and cardiac troponin $\mathrm{T}$ levels were used to detect the degree of cardiac damage. The levels of superoxide dismutase (SOD), malondialdehyde, catalase and total antioxidant capacity in the serum and heart were measured to observe the oxidative stress state of rats. Primary cardiomyocytes were cultured, and cell viability and reactive oxygen species (ROS) production were detected. Western blotting was used to detect the expression levels of SOD2, NOX2, pro/cleaved-caspase3 and $\mathrm{Bcl}-2 / \mathrm{Bax}$ in heart tissue and primary cardiomyocytes to verify the related signaling pathways. THP-treated rats showed a range of cardiac damage, including an abnormal ECG, echocardiography and myocardial enzymes. In the cellular experiments, cell viability decreased and ROS increased. However, this damage was alleviated after SchB treatment. Further studies demonstrated that SchB antagonized THP cardiotoxicity via its antioxidant effect. In conclusion, $\mathrm{SchB}$ protects the heart from THP damage in
\end{abstract}

Correspondence to: Professor Peng Pu or Professor Li Wen, Department of Cardiology, The First Affiliated Hospital of Chongqing Medical University, 1 Youyi Road, Yuanjiagang, Yuzhong, Chongqing 400042, P.R. China

E-mail: pp841103@sina.com

E-mail: 851557800th@sina.com

*Contributed equally

Key words: pirarubicin, schisandrin B, cardiotoxicity, oxidative stress rats, and the mechanism may be closely associated with its antioxidant effect.

\section{Introduction}

Pirarubicin (THP) is a doxorubicin analog that has gradually replaced doxorubicin in clinical practice (1). The antitumor effects of THP are more favorable than those of doxorubicin, with lower toxicity and fewer side effects (2). However, cardiotoxicity remains an issue (3). Different levels of cardiac damage have been reported in early THP treatment stages, and its long-term use may lead to irreversible cardiac damage and increased cardiovascular end events, thereby limiting its clinical applications $(4,5)$. Patients with tumors are often required to reduce or even stop THP therapy due to cardiac intolerance (6). Previous studies have shown that oxidative stress injury was implicated in THP-induced cardiac injury and was the initial step in cardiomyocyte apoptosis and necrosis, with effects including a reduced $\mathrm{Bcl}-2 / \mathrm{Bax}$ ratio and activation of the caspase family $(7,8)$. Other studies reported that reducing or even reversing oxidative stress injury helped prevent and treat THP-induced cardiac injury $(8,9)$.

Schisandrin B (SchB), which is derived from Schisandra chinensis, has very high biphenylcyclooctene lignin levels and has been clinically shown to improve antioxidant capacity levels and promote cell mitochondrial functions $(10,11)$. As a raw material, it may be added to functional foods, herbal dietary supplements, antiaging health care products and skin care products to protect the body from free radicals (12-14). Recent studies have also reported that long-term administration of low-dose SchB (Below normal treatment or modeling concentrations) increased the function and antioxidant capacity of mitochondria in the brain, heart, liver and skeletal muscle of young and old experimental rats $(12,15,16)$. Additionally, $\mathrm{SchB}$ administered to rats (a myocardial infarction model) protected cardiomyocytes from ischemia/reperfusion injury (17).

Therefore, we hypothesized that $\mathrm{SchB}$ could protect the heart from THP damage via antioxidant mechanisms. To the best of our knowledge, this hypothesis has not yet been previously confirmed in vivo or in vitro. Therefore, the current study investigated the antioxidant effects of SchB during THP-induced cardiac damage and preliminarily evaluated the key antioxidant mechanisms. 


\section{Materials and methods}

Materials. SchB and THP, purity $\geq 98 \%$, were purchased from Shanghai Aladdin Reagent Co., Ltd. Malondialdehyde (MDA; cat. no. A003-1-2), superoxide dismutase (SOD; cat. no. A001-3-2), catalase (CAT; cat. no. A007-1-1), total antioxidant capacity (T-AOC; cat. no. A015-1-2) and lactate dehydrogenase (LDH; cat. no. A020-2-2) test kits were obtained from Nanjing Jiancheng Biological Engineering Research Institute. Brain natriuretic peptide (BNP; cat. no. MB-1608A), creatine kinase MB (CK-MB; cat. no. MB-6930A) and cardiac troponin T (cTnT; cat. no. MB-7278A) test kits were purchased from Shanghai Meixuan Biological Science and Technology, Ltd. A reactive oxygen species (ROS) detection kit and Cell Counting Kit (CCK)-8 cell viability and toxicity detection kits were acquired from Biosharp Life Sciences. Antibodies against SOD2 (cat. no. 13141S), pro/cleaved caspase-3 (cat. nos. 14220S/9664S) and Bax (cat. no. 14796) were obtained from Cell Signaling Technology, Inc., and antibodies against NADPH oxidase 2 (NOX2; cat. no. 19013-1-AP) and Bcl-2 (cat. no. 26593-1-AP) were obtained from ProteinTech Group, Inc. All chemicals and reagents were of analytical grade.

\section{Animal experiments}

Animal model. This study was approved by the Animal Ethics Committee of The First Affiliated Hospital of Chongqing Medical University (CMU; approval no. 20195101). A total of 20 male Sprague Dawley (SD) rats (weight, 180-200 g; age, 6 weeks) were obtained from the CMU Experimental Animal Center. Rats were kept at a standard room temperature of $22 \pm 3^{\circ} \mathrm{C}$ with $45 \pm 10 \%$ humidity under a $12 \mathrm{~h}$ light/dark cycle. The animals were supplied with ad libitum standard laboratory food and tap water prior to experimentation. Rats were randomly distributed equally into four groups $(n=5$ in each group): Control (CON) group (normal diet for 7 weeks), SchB group (SchB-supplemented diet, $50 \mathrm{mg} / \mathrm{kg}$ for 7 weeks), THP group ( $3 \mathrm{mg} / \mathrm{kg}$ THP was injected via the caudal vein once a week with a normal diet for 7 weeks) and SchB + THP group ( $3 \mathrm{mg} / \mathrm{kg}$ THP was injected via the caudal vein once a week with an SchB-supplemented diet, $50 \mathrm{mg} / \mathrm{kg}$ for 7 weeks). The doses of THP and SchB were converted from the doses taken by patients clinically and according to our previous research (18-20). Rats in the CON and THP groups were fed an AIN-76A diet. The AIN-76A diet contained $\sim 5.2 \%$ fat (\% by weight, approx. all from corn oil). The SchB diet in the SchB group and SchB + THP group contained $\sim 0.5 \%$ SchB in AIN-76A feed. After conversion, $0.5 \%$ SchB in the diet $=50 \mathrm{mg} / \mathrm{kg}$ in rats. Similar feed processing and feeding schemes can be found in our previous studies $(21,22)$. AIN-76A feed and SchB feed processing were completed by Jiangsu Synergy Pharmaceutical Bioengineering Co., Ltd. Food consumption and body weight were measured twice a week.

Electrocardiogram (ECG) and Doppler echocardiography. At week 8, SD rats were anesthetized with inhaled isoflurane (2\%, the maintenance dose was also $2 \%)$. Three lead on ECG was recorded by a BL-420F biological function measurement system (Chengdu Taimeng Software Co., Ltd.). Doppler echocardiography was measured by using a Vivid E95 ultrasonic diagnostic apparatus (General Electric Company).

Sample collection and processing. Rats were sacrificed via cervical dislocation under anesthesia (inhalation of $2 \%$ isoflurane). Blood samples were collected from the abdominal aorta and centrifuged at $314 \mathrm{xg}$ for $30 \mathrm{~min}$ at $4^{\circ} \mathrm{C}$. The supernatant was stored in a refrigerator at $-80^{\circ} \mathrm{C}$. A cardiac tissue sample was then removed and stored at $-80^{\circ} \mathrm{C}$. The levels of $\mathrm{LDH}$, BNP, CK-MB, cTnT, SOD and MDA in serum were determined according to the instructions of the kits. A total of $\sim 100 \mathrm{mg}$ heart tissue was homogenized in normal saline at a ratio of 1:10 and then centrifuged in a low-temperature centrifuge at $1,250 \mathrm{xg}$ for $15 \mathrm{~min}$ at $4^{\circ} \mathrm{C}$. The supernatant was obtained to determine the contents of SOD, MDA and CAT and the T-AOC in accordance with the manufacturer's protocol.

Cell culture and treatment. The relevant extraction methods for primary cardiomyocytes have been described in our previous study (18). A total of 20 neonatal male SD rats (age, 1-3 days; weight, 5-6 g) were kept at a standard room temperature of $22 \pm 3^{\circ} \mathrm{C}$ with $45 \pm 10 \%$ humidity under a $12 \mathrm{~h}$ light/dark cycle at the CMU Experimental Animal Center. Animals were not fed and were immediately anesthetized and disinfected with $75 \%$ ethanol. The ventricular areas were quickly isolated under aseptic conditions. Blood clots, blood vessels and fat in the heart were removed, and the remaining tissue was washed clean, cut into chylous shapes $\left(1 \mathrm{~mm}^{3}\right)$, digested by trypsin + type II collagenase, filtered, centrifuged $\left(200 \mathrm{x} \mathrm{g}\right.$ at $26^{\circ} \mathrm{C}$ for $\left.5 \mathrm{~min}\right)$, suspended and seeded. Finally, primary rat cardiomyocytes were obtained by the differential adhesion method and seeded in six-well plates at a density of $70-80 \%$ (18). The obtained primary rat cardiomyocytes were further cultured for $24-48 \mathrm{~h}$ in DMEM (Gibco; Thermo Fisher Scientific, Inc.) with $10 \%$ FBS (PAN-Biotech $\mathrm{GmbH}$ ) and $2 \%$ penicillin/streptomycin at $37.5^{\circ} \mathrm{C}, \mathrm{pH} 7.2-7.4$ and $95 \%$ air $+5 \% \mathrm{CO}_{2}$. Cardiomyocytes were then treated $\left(37.5^{\circ} \mathrm{C}\right)$ according to the following methods: Normal group (CON), SchB group (SchB, $50 \mu \mathrm{M}, 14 \mathrm{~h}$ ), THP group (THP, $10 \mu \mathrm{M}, 12 \mathrm{~h}$ ), and THP + SchB coculture group (SchB, $50 \mu \mathrm{M}, 2 \mathrm{~h}$; SchB $50 \mu \mathrm{M}+$ THP $10 \mu \mathrm{M}, 12 \mathrm{~h}$ ). Approximately every 15 neonatal rat cardiomyocytes were placed into a standard six-well plate. A total of $\sim 4$ six-well plates were used, with an average of six wells in each group. Cell viability and oxidative stress in each group were detected according to the instructions of the CCK-8 and ROS kits (23).

Western blotting. Heart tissue and primary rat cardiomyocytes were lysed in RIPA lysis buffer with $1 \%$ protease inhibitor (Beyotime Institute of Biotechnology). A BCA kit was used to determine the protein concentration in the supernatant (Beyotime Institute of Biotechnology). In total, $\sim 50 \mu \mathrm{g}$ heart tissue lysate or $20 \mu \mathrm{g}$ cell lysate was used for $12 \%$ SDS-PAGE, and proteins were then transferred to an FL membrane (MilliporeSigma) at $4^{\circ} \mathrm{C}$ for $1.5 \mathrm{~h}$. After blocking with 5\% non-fat milk powder (Beyotime Institute of Biotechnology) at room temperature for $1.5 \mathrm{~h}$, the following primary antibodies were added and incubated overnight at $4^{\circ} \mathrm{C}$ : SOD2 $(1: 1,000)$, pro/cleaved caspase-3 (1:1,000), Bax (1:1,000), NOX2 $(1: 2,000)$ and Bcl-2 (1:1,000) were. Subsequently, HRP-conjugated goat anti-rabbit IgG $(\mathrm{H}+\mathrm{L})$ secondary antibodies $(1: 10,000$; 
A
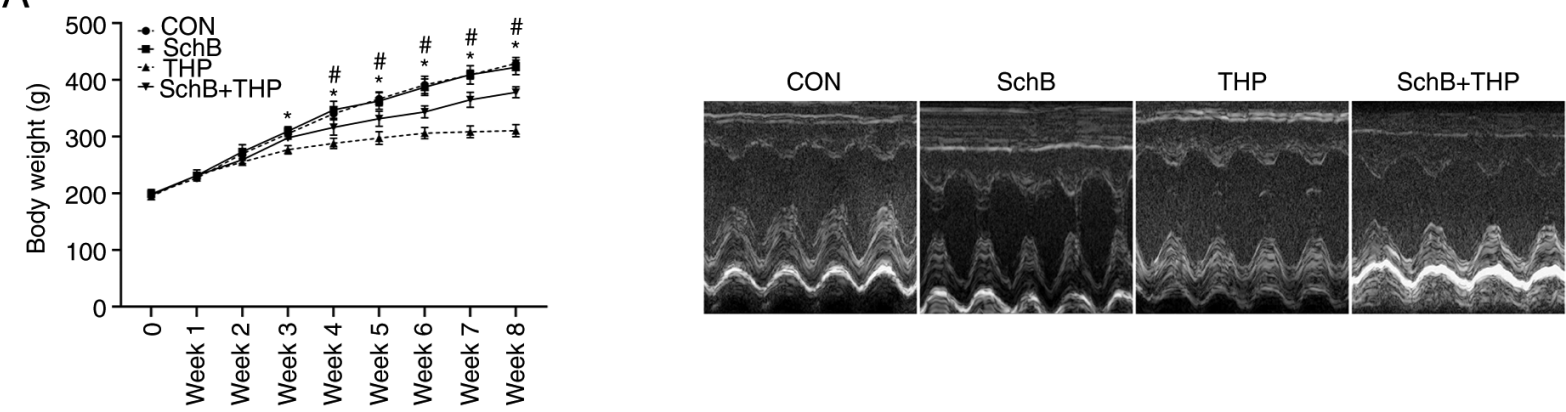

B
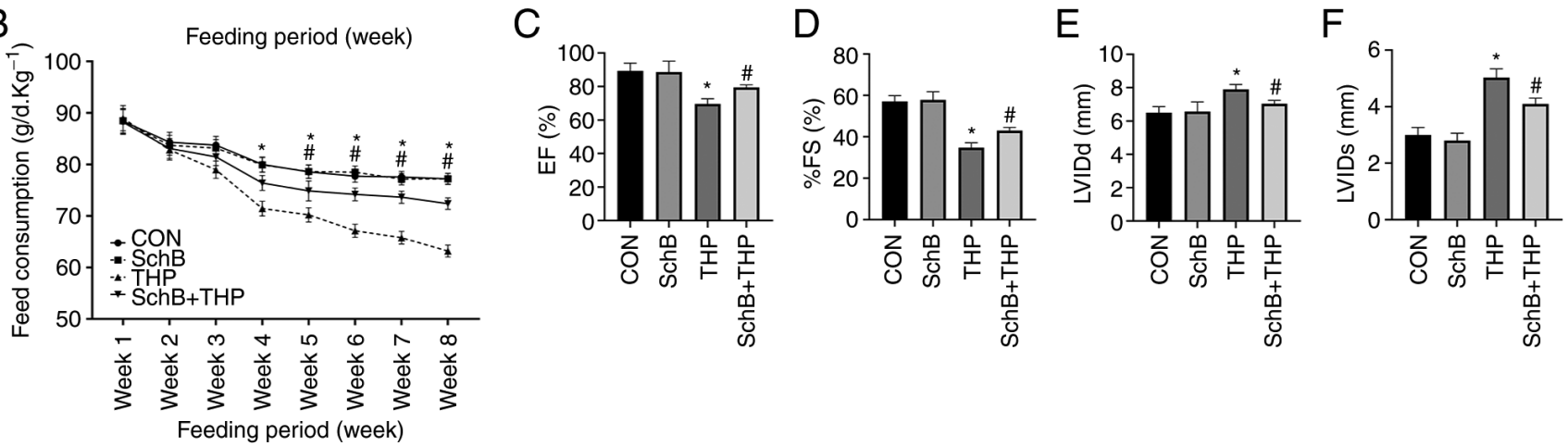

Figure 1. SchB improves THP-induced body weight, food intake and echocardiographic changes in rats. (A) Body weights and (B) food intake of THP-treated rats decreased in the third and fourth weeks. (C) EF and (D) \%FS decreased, and (E) LVIDd and (F) LVIDs increased in THP-treated rats. After treatment with $\mathrm{SchB}$, the aforementioned changes were effectively alleviated. All values are presented as the mean $\pm \mathrm{SD}$. ${ }^{*} \mathrm{P}<0.05$ vs. CON; ${ }^{\#} \mathrm{P}<0.05$ vs. THP. EF, left ventricular ejection fraction; FS, left ventricular shortening fraction; LVIDd, left ventricular internal diameter at end-diastole; LVIDs, left ventricular internal diameter at end-systole; CON, control; SchB, schisandrin B; THP, pirarubicin.

Thermo Fisher Scientific, Inc.; cat. no. 31460) were added and incubated at room temperature for $1.5 \mathrm{~h}$. The western blotting results were analyzed using BeyoECL Plus (Beyotime Institute of Biotechnology) and Image Lab software (version 5.2.1; Bio-Rad Laboratories, Inc.). The specific protein expression levels were normalized to that of GAPDH.

Statistical analysis. Data are presented as the mean \pm standard deviation $(n=3)$ and statistical analyses were performed using SPSS Statistics 26 (IBM Corp.). The significance of differences between groups was analyzed statistically using one or two-way ANOVA, followed by Tukey's multiple-comparison post hoc test. $\mathrm{P}<0.05$ was considered to indicate a statistically significant difference.

\section{Results}

SchB improves THP-induced body weight, food intake and echocardiographic changes in rats. The body weights (Fig. 1A) and food intake (Fig. 1B) of THP-administered rats began to decrease in the third and fourth weeks. However, significant improvements in the aforementioned changes were observed after treatment with SchB. Similarly, THP caused echocardiographic damage in rats, such as a decreased left ventricular ejection fraction (Fig. 1C), decreased left ventricular fractional shortening (Fig. 1D), increased left ventricular internal diameter (LVID) at end-diastole (Fig. 1E) and an increased LVID at end-systole (Fig. 1F). After treatment with SchB, the aforementioned changes were effectively alleviated.
SchB effectively improves THP-induced myocardial injury in rats. THP also caused myocardial injury in rats, including increased $\mathrm{R}$ waves (Fig. 2A) and T waves (Fig. 2B), decreased S waves (Fig. 2C) and prolonged QT intervals (Fig. 2D). Similarly, the serum markers of myocardial injury in rats were also abnormal, including increased LDH (Fig. 2E), BNP (Fig. 2F), CK-MB (Fig. 2G) and cTnT (Fig. 2H). After SchB treatment, the aforementioned changes were significantly improved (Fig. 2A-H).

SchB attenuates THP-induced oxidative stress in rats. The MDA content (Fig. 3A and E) was increased and the SOD content (Fig. 3B and F) was decreased in both serum and heart tissue of the THP group. Similarly, reductions were also detected with regards to CAT and T-AOC in the serum and heart. Moreover, SchB improved the THP-induced increase in MDA and the decrease in SOD, CAT (Fig. 3C and G) and T-AOC (Fig. 3D and H), indicating that SchB improved the antioxidant capacity of rats.

Subsequently, the expression of oxidative stress markers and related downstream proteins in heart tissue were evaluated. As shown in Fig. 4B, the expression levels of SOD2, pro-caspase-3 and $\mathrm{Bcl}-2 / \mathrm{Bax}$ were decreased, while the expression levels of NOX 2 and cleaved-caspase-3 were increased.

However, after treatment with SchB, the aforementioned changes were significantly improved, as shown by the semi-quantitative analyses (Fig. 4C).

SchB improves myocardial tissue changes induced by THP in rats. As shown in Fig. 4A, the myocardial cells in the THP group were disordered, the intercellular space was increased 

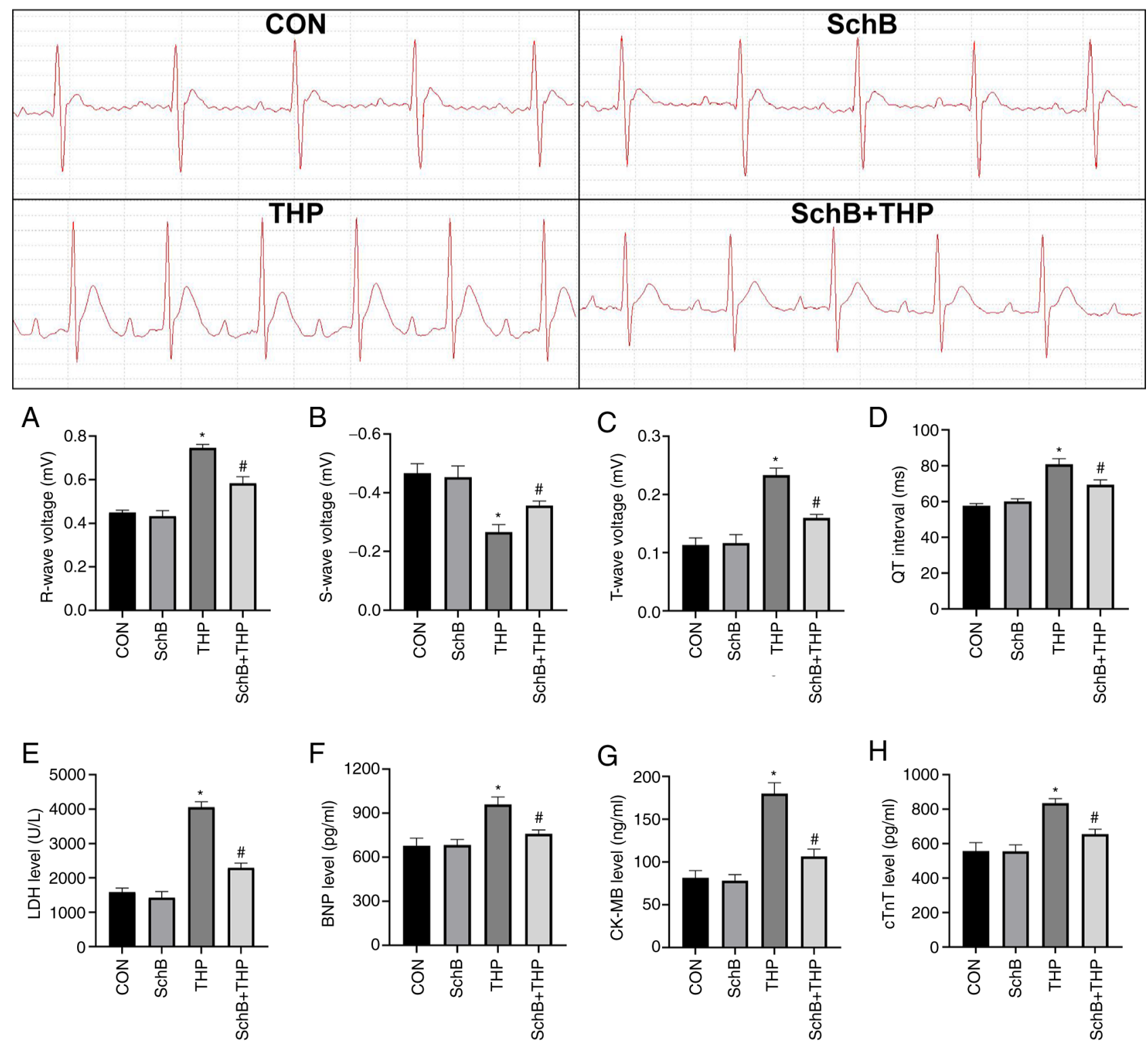

Figure 2. SchB effectively improves THP-induced myocardial injury in rats. The (A) R wave and (B) S wave increased; the (C) $\mathrm{T}$ wave decreased; and the (D) QT interval was prolonged in THP-treated rats. Similarly, (E) LDH, (F) BNP, (G) CK-MB and (H) cTnT increased in the serum of THP-treated rats. After $\mathrm{SchB}$ treatment, the aforementioned changes were significantly improved. All values are presented as the mean $\pm \mathrm{SD}$. ${ }^{*} \mathrm{P}<0.05$ vs. CON; ${ }^{~} \mathrm{P}<0.05$ vs. THP. $\mathrm{LDH}$, lactate dehydrogenase; BNP, brain natriuretic peptide; CK-MB, creatine kinase MB; cTnT, and cardiac troponin T; CON, control; SchB, schisandrin B; THP, pirarubicin.

and the myocardial tissue was calcified or denatured. However, these changes in the heart were alleviated after SchB treatment.

SchB attenuates THP-induced oxidative stress in primary cardiomyocytes. As shown in Fig. 5A and B, THP reduced primary cardiomyocyte viability and increased ROS levels. Similarly, the expression levels of oxidative stress markers and related downstream proteins in primary cardiomyocytes were detected. The results suggested that the expression levels of SOD2, pro-caspase-3 and Bcl-2/Bax were decreased, while the expression levels of NOX2 and cleaved-caspase- 3 were increased in the THP group (Fig. 5C).

However, after treatment with SchB, the aforementioned changes were significantly improved, as shown by the semi-quantitative analyses (Fig. 5D).

\section{Discussion}

At a $50-\mathrm{mg} / \mathrm{kg}$ dose, $\mathrm{SchB}$ displayed novel and promising effects by improving THP-induced cardiotoxicity in rats and produced considerable improvements in a series of cardiac injury manifestations. Consistent with our hypothesis, the key to improving THP-induced cardiac injury by SchB was combatting increased oxidative stress. Thus, the current study provided an improved understanding of the pharmacological effects of SchB and novel insights for the development of effective natural products to prevent THP cardiotoxicity.

At present, SchB has shown some achievements in the study of the cardiotoxicity of anthracycline antitumor drugs. However, the data are relatively old and all focus on adriamycin $(24,25)$. Adriamycin has been gradually abandoned in clinical practice, while other corresponding anthracycline 
A

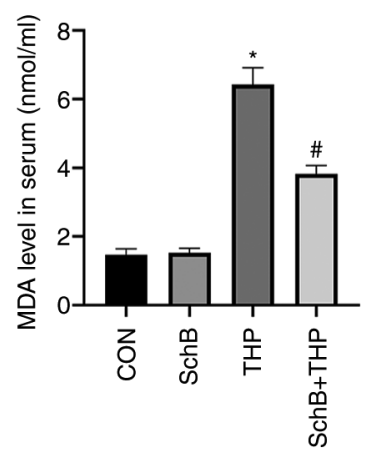

E

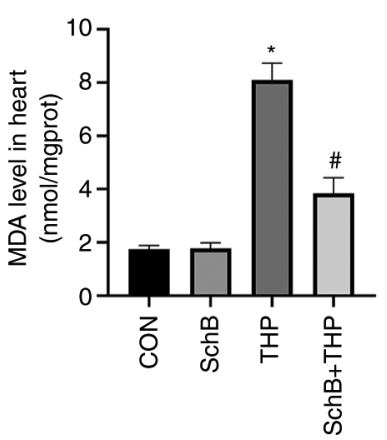

B

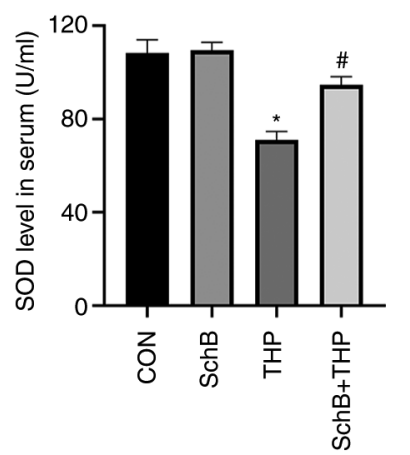

F

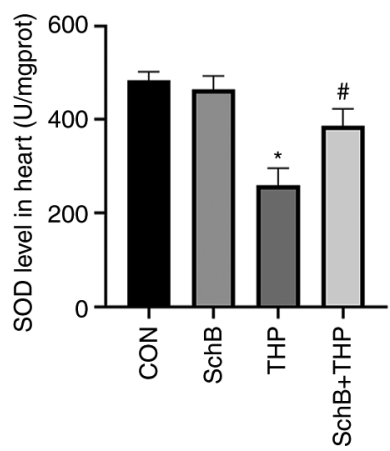

C

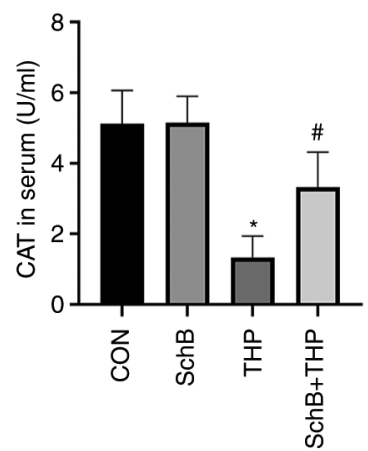

G

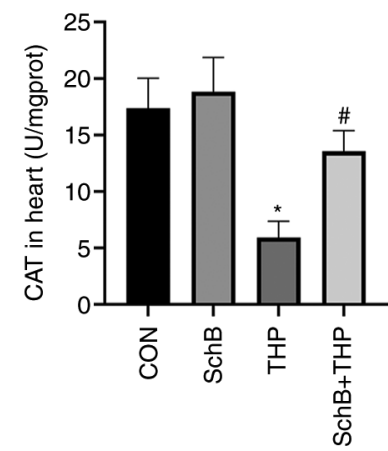

D

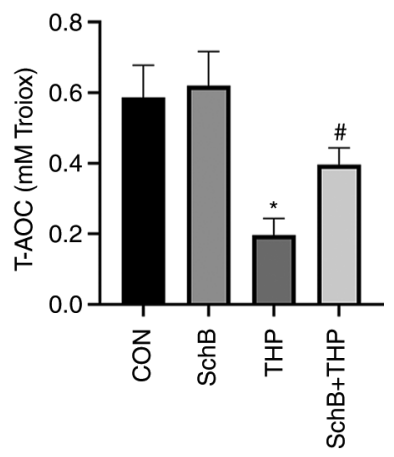

$\mathrm{H}$

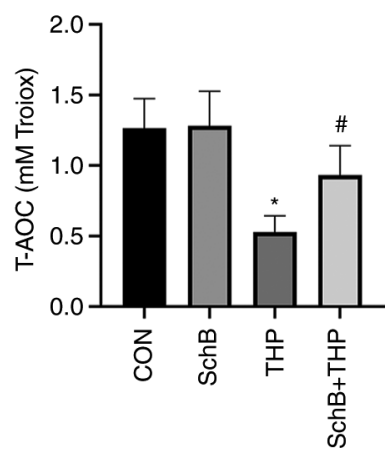

Figure 3. SchB attenuates THP-induced oxidative stress in rats. (A and E) MDA content increased and the (B and F) SOD content decreased in both serum and heart tissue. SchB also increased the THP-induced decrease in $(\mathrm{C}$ and $\mathrm{G}) \mathrm{CAT}$ and $(\mathrm{D}$ and $\mathrm{H}) \mathrm{T}$-AOC. All values are presented as the mean \pm SD ${ }^{*} \mathrm{P}<0.05$ vs. CON; "P<0.05 vs. THP. SOD, superoxide dismutase; MDA, malondialdehyde; CAT, catalase; T-AOC, total antioxidant capacity; CON, control; SchB, schisandrin B; THP, pirarubicin.

A

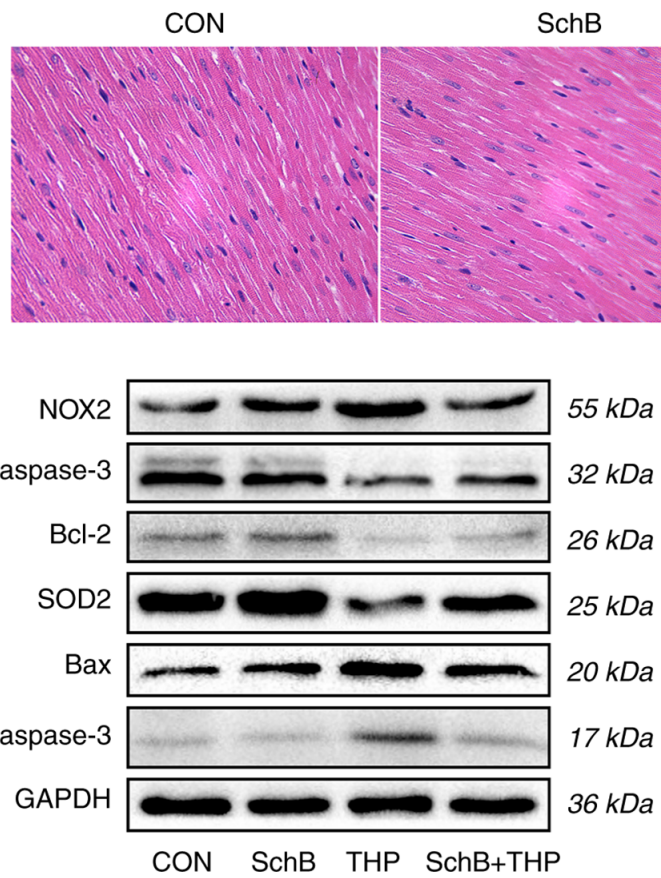

THP

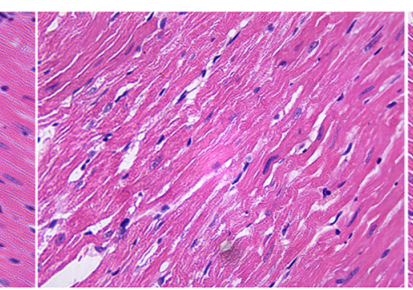

SchB+THP

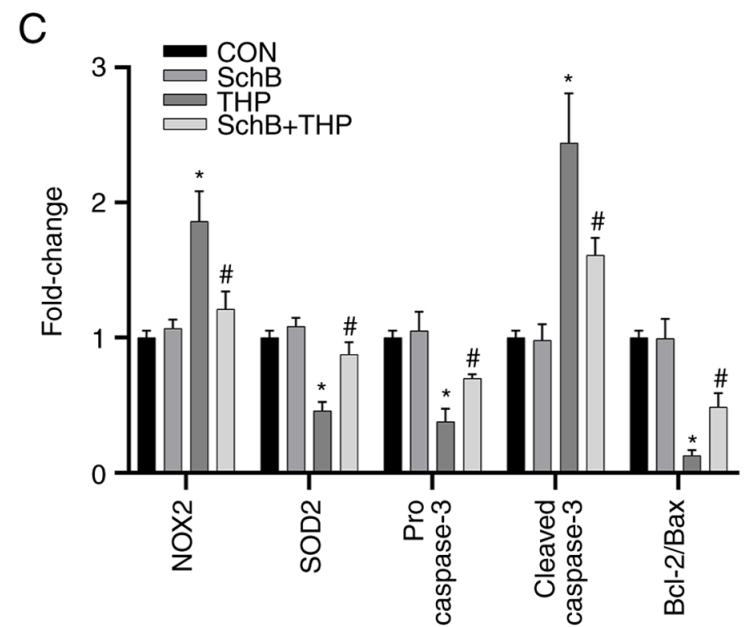

Figure 4. SchB improves myocardial tissue changes induced by THP in rats. (A) The myocardial cells in the THP group were disordered, the intercellular space was increased and the myocardial tissue was calcified or denatured. However, these changes in the heart were alleviated after SchB treatment (magnification, x200). (B) The expression levels of SOD2, pro-caspase-3 and Bcl-2/Bax decreased, while the expression levels of NOX2 and cleaved-caspase-3 increased. However, after treatment with SchB, the aforementioned changes were significantly improved. (C) Semi-quantitative analysis of western blotting results. Magnification, $x 200$. All values are presented as the mean \pm SD. ${ }^{P}<<0.05$ vs. CON; ${ }^{*} \mathrm{P}<0.05$ vs. THP. SOD, superoxide dismutase; NOX, NADPH oxidase; CON, control; SchB, schisandrin B; THP, pirarubicin. 
A

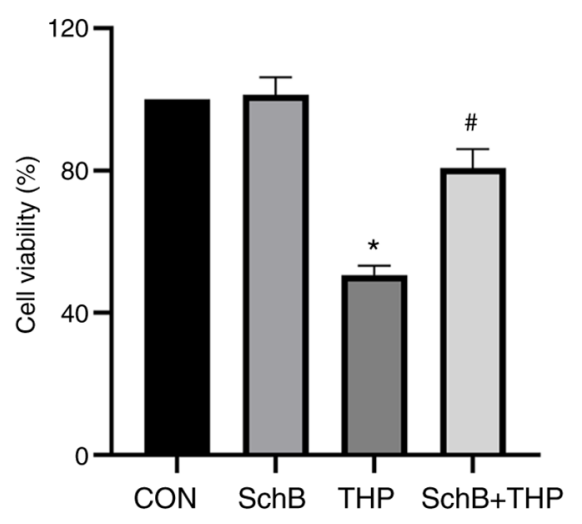

B

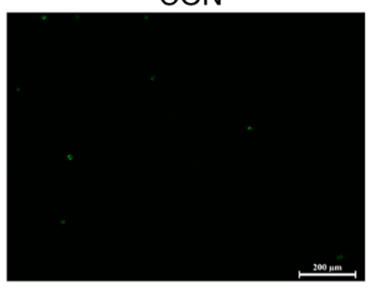

THP

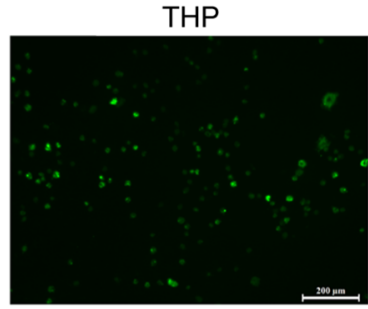

$\operatorname{SchB}$

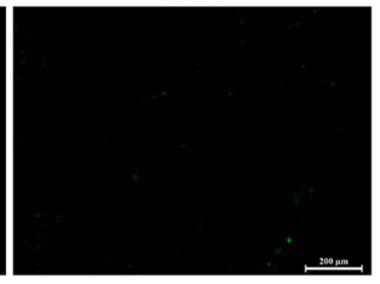

SchB+THP

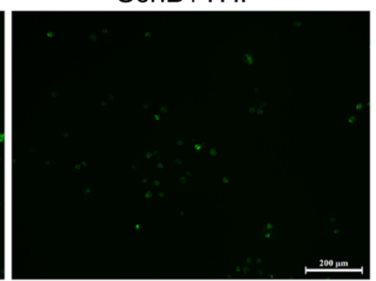

C

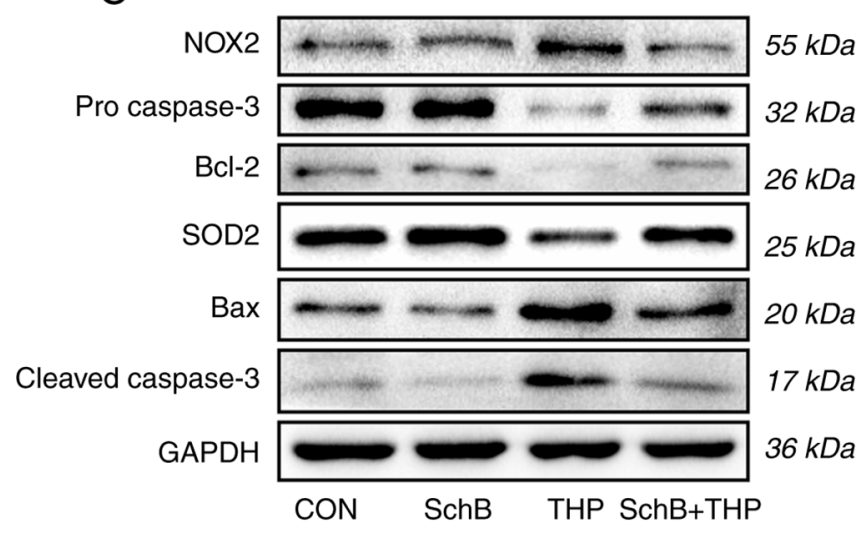

D

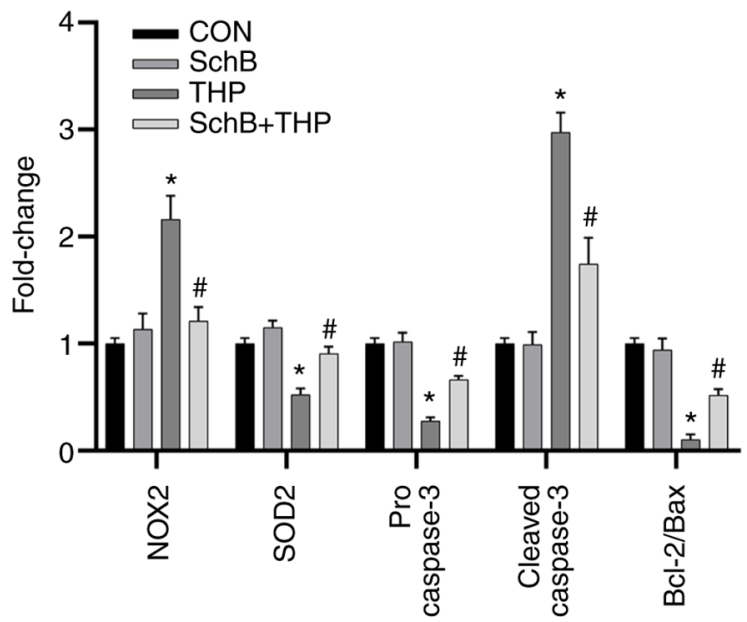

Figure 5. SchB attenuates THP-induced oxidative stress in primary cardiomyocytes. THP reduced H9C2 (A) cardiomyocyte viability and (B) increased reactive oxygen species. Scale bar, $200 \mu \mathrm{m}$. (C) Similarly, the expression levels of SOD2, pro-caspase-3 and Bcl-2/Bax decreased, while the expression levels of NOX2 and cleaved-caspase-3 increased. However, after treatment with SchB, the aforementioned changes were significantly improved. (D) Semi-quantitative analysis of western blotting results. All values are presented as the mean \pm SD. ${ }^{*} \mathrm{P}<0.05$ vs. CON; ${ }^{~ P}<0.05$ vs. THP. SOD, superoxide dismutase; NOX, NADPH oxidase; CON, control; SchB, schisandrin B; THP, pirarubicin.

antitumor drugs, such as THP, are more widely used. THP is an anthracycline antitumor drug; however, cardiotoxicity issues have limited its clinical application (3). Often, patients with cancer must reduce doses or even stop therapy due to cardiac intolerance (6). Echocardiography and ECG are commonly used as cardiac function test methods to monitor patients receiving anthracycline drugs $(26,27)$. Without intervention, patients or animals on long-term anthracyclines may incur some level of echocardiography and ECG damage, which is consistent with our present observations $(26,27)$. In addition, myocardial injury serum markers, such as CK-MB, cTnT and BNP, also reflect damage to cardiac function and structure $(28,29)$.

Cardiotoxicity induced by anthracyclines is closely associated with oxidative stress (30). High ROS levels activate cytotoxic signals leading to DNA damage, mitochondrial dysfunction, altered protein synthesis and calcium overload, eventually leading to irreversible cardiomyocyte damage $(30,31)$. The end-product of oxidation is
MDA, which causes cross-linking and polymerization of proteins, nucleic acids and other key macromolecules to induce cytotoxicity (32), thereby affecting the activity of in vitro mitochondrial respiratory chain complexes and key enzymes in mitochondria (22). SOD is also regarded as a major destroyer of oxygen free radicals in the body, which resists, blocks and recovers the damage caused by oxygen free radicals and repairs damaged cells over time $(33,34)$. Similarly, in the current THP model, MDA levels increased, and SOD activity, CAT levels and the T-AOC decreased in both serum and heart tissue, suggesting that THP induced abnormal increases in oxidative stress in the model. NOX is the main ROS source in cardiovascular systems, and NOX2 is the earliest NOX subtype that transfers NADPH electrons to molecular oxygen, generating superoxide anions $\left(\mathrm{O}^{2-}\right)$ and inducing disease (35-37). Increased NOX2 expression in the rat heart suggested that THP induced ROS overproduction by activating NOX2 expression. Similar results were obtained in vitro. 
Another important finding of the present study was that SchB had promising protective effects on THP-induced cardiac injury; these injuries were improved, and SchB reduced oxidative stress levels in rats and primary cardiomyocytes. Consistent with other studies $(12,38), \mathrm{SchB}$ increased SOD levels, inhibited lipid peroxidation, reduced the release of LDH, MDA and ROS, and directly scavenged free radicals. These scavenging effects on oxygen free radicals were a significant feature of SchB function, and, critically, these effects on hydroxyl free radicals were greater than those of vitamin $\mathrm{C}$ at the same concentration (39). Excessive oxidative stress in the heart and myocardial cells also induces apoptosis and eventually leads to myocardial cell death (40). Consistent with the present results, excessive ROS production led to activation of the caspase protein family and decreased the Bcl-2/Bax ratio, which eventually led to increased cardiomyocyte apoptosis and affected normal heart function $(41,42)$. SchB effectively reversed these harmful changes both in vivo and in vitro.

The present study confirmed that the cardiovascular protective effects of SchB were dependent on reducing oxidative stress levels. The current results provided evidence that $\mathrm{SchB}$, as a natural molecule, exerted strong cardiovascular protective effects and highlighted its key potential antioxidant stress mechanisms. However, at the end of the experiment, representative images of rats in each group were not captured, and the general state of rats was not observed. In addition, how SchB regulated oxidative stress and some specific mechanisms remains unclear. Therefore, further studies are required to clarify the potential role of SchB as a new and effective antioxidant drug for drug-induced cardiovascular disease and other cardiovascular diseases.

In conclusion, the present study identified that SchB effectively improved heart injury caused by THP, which was closely associated with its strong antioxidant capacity. Based on this evidence, SchB may be a promising new drug for the prevention and treatment of cardiovascular disease caused by abnormal increases in oxidative stress mediated by drugs or other causes. A clinical study by the authors will be conducted soon, but at present the basic research is ongoing and will explore other mechanisms and therapeutic targets.

\section{Acknowledgements}

Not applicable.

\section{Funding}

The study was supported by a research grant from the National Natural Science Foundation of China (grant no. 31501097).

\section{Availability of data and materials}

The datasets used and/or analyzed during the current study are available from the corresponding author on reasonable request.

\section{Authors' contributions}

HT and JZ conceived and designed the experiments. PP and LW implemented experimental improvements. HT and JZ acquired and analyzed the data. HT, PP, LW and RF analyzed and interpreted the data. HT, PP and RF drafted manuscript and critically revised it for important intellectual content. All authors confirm the authenticity of all the raw data. All authors read and approved the final version of the manuscript.

\section{Ethics approval and consent to participate}

This study was approved by the Animal Ethics Committee of The First Affiliated Hospital of Chongqing Medical University (approval no. 20195101).

\section{Patient consent for publication}

Not applicable.

\section{Competing interests}

The authors declare that they have no competing interests.

\section{References}

1. Minotti G, Recalcati S, Menna P, Salvatorelli E, Corna G and Cairo G: Doxorubicin cardiotoxicity and the control of iron metabolism: Quinone-dependent and independent mechanisms. Methods Enzymol 378: 340-361, 2004.

2. Weiss RB: The anthracyclines: Will we ever find a better doxorubicin? Semin Oncol 19: 670-686, 1992.

3. Maayah ZH, Abdelhamid G, Elshenawy OH, El-Sherbeni AA, Althurwi HN, McGinn E, Dawood D, Alammari AH and El-Kadi AOS: The role of soluble epoxide hydrolase enzyme on daunorubicin-mediated cardiotoxicity. Cardiovasc Toxicol 18: 268-283, 2018.

4. Skrypnyk I, Maslova G, Lymanets T and Gusachenko I: L-arginine is an effective medication for prevention of endothelial dysfunction, a predictor of anthracycline cardiotoxicity in patients with acute leukemia. Exp Oncol 39: 308-311, 2017.

5. Nicolazzi MA, Carnicelli A, Fuorlo M, Scaldaferri A, Masetti R, Landolfi R and Favuzzi AMR: Anthracycline and trastuzumab-induced cardiotoxicity in breast cancer. Eur Rev Med Pharmacol Sci 22: 2175-2185, 2018.

6. Bartlett JJ, Trivedi PC and Pulinilkunnil T: Autophagic dysregulation in doxorubicin cardiomyopathy. J Mol Cell Cardiol 104: 1-8, 2017.

7. Afsar T, Razak S, Batoo KM and Khan MR: Acacia hydaspica R. Parker prevents doxorubicin-induced cardiac injury by attenuation of oxidative stress and structural Cardiomyocyte alterations in rats. BMC Complement Altern Med 17: 554, 2017.

8. Songbo M, Lang H, Xinyong C, Bin X, Ping Z and Liang S: Oxidative stress injury in doxorubicin-induced cardiotoxicity. Toxicol Lett 307: 41-48, 2019.

9. Zhao L, Qi Y, Xu L, Tao X, Han X, Yin L and Peng J: MicroRNA-140-5p aggravates doxorubicin-induced cardiotoxicity by promoting myocardial oxidative stress via targeting Nrf2 and Sirt2. Redox Biol 15: 284-296, 2018.

10. Lu TL, Wu XY, Song Y, Chen H, Xu B, Zhou Y, Huang ZJ, Sun Y and Mao CQ: Effect of acupuncture on target tissue distribution of Schisandra lignans. Acupunct Med 31: 207-213, 2013.

11. Wu Y, Li ZC, Yao LQ, Li M and Tang M: Schisandrin B alleviates acute oxidative stress via modulation of the Nrf2/Keap1-mediated antioxidant pathway. Appl Physiol Nutr Metab 44: 1-6, 2019.

12. Lam PY and Ko KM: Schisandrin B as a hormetic agent for preventing age-related neurodegenerative diseases. Oxid Med Cell Longev 2012: 250825, 2012.

13. Lam PY, Yan CW, Chiu PY, Leung HY and Ko KM: Schisandrin $B$ protects against solar irradiation-induced oxidative stress in rat skin tissue. Fitoterapia 82: 393-400, 2011.

14. Nasser MI, Zhu S, Chen C, Zhao M, Huang H and Zhu P: A comprehensive review on schisandrin $\mathrm{B}$ and its biological properties. Oxid Med Cell Longev 2020: 2172740, 2020.

15. Zhu H, Zhang X, Guan J, Cui B, Zhao L and Zhao X: Pharmacokinetics and tissue distribution study of schisandrin $\mathrm{B}$ in rats by ultra-fast liquid chromatography with tandem mass spectrometry. J Pharm Biomed Anal 78-79: 136-140, 2013. 
16. Jiang EP, Li H, Yu CR, Yu CY, Jing S, Sun HX, Wang CM, Fan XT, Chen JG and Wang S: Schisandrin B protects PC12 cells against oxidative stress of neurodegenerative diseases. Neuroreport 26: 360-366, 2015.

17. Zhao X, Xiang Y, Cai C, Zhou A, Zhu N and Zeng C: Schisandrin B protects against myocardial ischemia/reperfusion injury via the PI3K/Akt pathway in rats. Mol Med Rep 17: 556-561, 2018.

18. Shi H, Zeng Q, Wei Y, Yang H, Tang H, Wang D, Pu P and Feng R: Canagliflozin is a potential cardioprotective drug but exerts no significant effects on pirarubicin-induced cardiotoxicity in rats. Mol Med Rep 24: 703, 2021.

19. Chen N and Ko M: Schisandrin B-induced glutathione antioxidant response and cardioprotection are mediated by reactive oxidant species production in rat hearts. Biol Pharm Bull 33: 825-829, 2010.

20. Miller AA and Salewski E: Prospects for pirarubicin. Med Pediatr Oncol 22: 261-688, 1994.

21. Tang $\mathrm{H}$, Zeng Q, Tang $\mathrm{T}$, Wei $\mathrm{Y}$ and $\mathrm{Pu} \mathrm{P}$ : Kaempferide improves glycolipid metabolism disorder by activating PPAR $\gamma$ in high-fat-diet-fed mice. Life Sci 270: 119133, 2021.

22. Tang $\mathrm{H}$, Zeng Q, Ren N, Wei Y, He Q, Chen M and Pu P: Kaempferide improves oxidative stress and inflammation by inhibiting the TLR4/I $\kappa \mathrm{B} \alpha / \mathrm{NF}-\kappa \mathrm{B}$ pathway in obese mice. Iran J Basic Med Sci 24: 493-498, 2021.

23. Xu J, Liu D, Niu H, Zhu G, Xu Y, Ye D, Li J and Zhang Q: Resveratrol reverses Doxorubicin resistance by inhibiting epithelial-mesenchymal transition (EMT) through modulating PTEN/Akt signaling pathway in gastric cancer. J Exp Clin Cancer Res 36: 19, 2017.

24. Li L, Pan Q, Han W, Liu Z, Li L and Hu X: Schisandrin B prevents doxorubicin-induced cardiotoxicity via enhancing glutathione redox cycling. Clin Cancer Res 13: 6753-6760, 2007.

25. Xu Y, Liu Z, Sun J, Pan Q, Sun F, Yan Z and Hu X: Schisandrin $B$ prevents doxorubicin-induced chronic cardiotoxicity and enhances its anticancer activity in vivo. PLoS One 6: e28335, 2011.

26. Anqi Y, Yu Z, Mingjun X, Xiaoli K, Mengmeng L, Fangfang L and Mei Z: Use of echocardiography to monitor myocardial damage during anthracycline chemotherapy. Echocardiography 36 : 495-502, 2019

27. Saidi A and Alharethi R: Management of chemotherapy induced cardiomyopathy. Curr Cardiol Rev 7: 245-249, 2011.

28. Simões R, Silva LM, Cruz A, Fraga VG, de Paula Sabino A and Gomes KB: Troponin as a cardiotoxicity marker in breast cancer patients receiving anthracycline-based chemotherapy: A narrative review. Biomed Pharmacother 107: 989-996, 2018.

29. Cardinale D, Sandri MT, Colombo A, Colombo N, Boeri M, Lamantia G, Civelli M, Peccatori F, Martinelli G, Fiorentini C and Cipolla CM: Prognostic value of troponin I in cardiac risk stratification of cancer patients undergoing high-dose chemotherapy. Circulation 109: 2749-2754, 2004.

30. Cappetta D, De Angelis A, Sapio L, Prezioso L, Illiano M, Quaini F, Rossi F, Berrino L, Naviglio S and Urbanek K: Oxidative stress and cellular response to doxorubicin: A common factor in the complex milieu of anthracycline cardiotoxicity. Oxid Med Cell Longev 2017: 1521020, 2017.
31. Farías JG, Molina VM, Carrasco RA, Zepeda AB, Figueroa E, Letelier P and Castillo RL: Antioxidant therapeutic strategies for cardiovascular conditions associated with oxidative stress. Nutrients 9: 966, 2017.

32. Yu Y and Zheng G: Troxerutin protects against diabetic cardiomyopathy through NF- $\kappa \mathrm{B} / \mathrm{AKT} / \mathrm{IRS} 1$ in a rat model of type 2 diabetes. Mol Med Rep 15: 3473-3478, 2017.

33. Huang ZW, Liu N, Li D, Zhang HY, Wang Y, Liu Y, Zhang LL and Ju XL: Angiopoietin-1 modified human umbilical cord mesenchymal stem cell therapy for endotoxin-induced acute lung injury in rats. Yonsei Med J 58: 206-216, 2017.

34. Sun HL, Peng ML, Lee SS, Chen CJ, Chen WY, Yang ML and Kuan YH: Endotoxin-induced acute lung injury in mice is protected by 5,7-dihydroxy-8-methoxyflavone via inhibition of oxidative stress and HIF-1 $\alpha$. Environ Toxicol 31: 1700-1709, 2016.

35. Gray SP and Jandeleit-Dahm KA: The role of NADPH oxidase in vascular disease - hypertension, atherosclerosis and stroke. Curr Pharm Des 21: 5933-5944, 2015.

36. Parajuli N, Patel VB, Wang W, Basu R and Oudit GY: Loss of NOX2 (gp91phox) prevents oxidative stress and progression to advanced heart failure. Clin Sci (Lond) 127: 331-340, 2014

37. Manuneedhi Cholan P, Cartland SP and Kavurma MM: NADPH oxidases, angiogenesis, and peripheral artery disease. Antioxidants (Basel) 6: 56, 2017.

38. Xin DQ, Hu ZM, Huo HJ, Yang XJ, Han D, Xing WH, Zhao Y and Qiu QH: Schisandrin B attenuates the inflammatory response, oxidative stress and apoptosis induced by traumatic spinal cord injury via inhibition of p53 signaling in adult rats. Mol Med Rep 16: 533-538, 2017.

39. Kim SR, Lee MK, Koo KA, Kim SH, Sung SH, Lee NG, Markelonis GJ, Oh TH, Yang JH and Kim YC: Dibenzocyclooctadiene lignans from Schisandra chinensis protect primary cultures of rat cortical cells from glutamate-induced toxicity. J Neurosci Res 76: 397-405, 2004.

40. Zhang X, Hu C, Kong CY, Song P, Wu HM, Xu SC, Yuan YP, Deng W, Ma ZG and Tang QZ: FNDC5 alleviates oxidative stress and cardiomyocyte apoptosis in doxorubicin-induced cardiotoxicity via activating AKT. Cell Death Differ 27: 540-55, 2020.

41. Wu Y, Wang B, Xu H, Tang L, Li Y, Gong L, Wang Y and Li W: Probiotic Bacillus attenuates oxidative stress-induced intestinal injury via p38-mediated autophagy. Front Microbiol 10: 2185, 2019.

42. Wang M, Meng XB, Yu YL, Sun GB, Xu XD, Zhang XP, Dong X, Ye JX, Xu HB, Sun YF and Sun XB: Elatoside C protects against hypoxia/reoxygenation-induced apoptosis in $\mathrm{H} 9 \mathrm{c} 2$ cardiomyocytes through the reduction of endoplasmic reticulum stress partially depending on STAT3 activation. Apoptosis 19: 1727-1735, 2014.

This work is licensed under a Creative Commons Attribution-NonCommercial-NoDerivatives 4.0 International (CC BY-NC-ND 4.0) License. 